\title{
Influence of humidity on granular packings with moving walls
}

\author{
Y. Bertho*, Th. Brunet ${ }^{\dagger}$, F. Giorgiutti-Dauphiné and J.-P. Hulin \\ Laboratoire FAST, UMR CNRS 7608, Bât. 502, \\ Université Paris XI, 91405 Orsay Cedex, France
}

\begin{abstract}
A significant dependence on the relative humidity $H$ for the apparent mass $\left(M_{\text {app }}\right)$ measured at the bottom of a granular packing inside a vertical tube in relative motion is demonstrated experimentally. While the predictions of Janssen's model are verified for all values of $H$ investigated $(25 \% \leq H \leq$ $80 \%), M_{\text {app }}$ increases with time towards a limiting value at high relative humidities $(H \geq 60 \%)$ but remains constant at lower ones $(H=25 \%)$. The corresponding Janssen length $\lambda$ is nearly independent of the tube velocity for $H \geq 60 \%$ but decreases markedly for $H=25 \%$. Other differences are observed on the motion of individual beads in the packing: for $H=25 \%$, they are almost motionless while the mean particle fraction of the packing remains constant; for $H \geq 60 \%$ the bead motion is much more significant and the mean particle fraction decreases. The dependence of these results on the bead diameter and their interpretation in terms of the influence of capillary forces are discussed.
\end{abstract}

PACS numbers: 45.70.-n, 68.08.Bc,81.05.Rm

Dense granular flows in vertical channels are encountered in many industrial processes and often display intermittency or blockage effects representing important practical problems [1, 2, 3]. These effects depend on the force distribution in the moving grain packing and on its interaction with the walls: these depend in turn on the relative humidity $H$ of the atmosphere. In static packings, capillary forces strongly influence, for instance, the stability of sand piles as a function of $H[4,5,6,6,6,8,9]$. In the present work, the influence of humidity on force transmission in a granular packing inside a vertical tube in relative motion is analyzed from variations of its apparent weight measured at the bottom of the packing.

Stress transmission in static or quasistatic granular packings has been frequently investigated theoretically and experimentally 10, 11, 12, 13, 14, 16, 17, 18, 19? ] following the pioneering work and Janssen 20]. This latter study and subsequent ones predict that the vertical stress at the bottom of a grain packing inside a vertical cylindrical tube reaches exponentially a limiting value as the height of the packing increases. This is due to the redirection of vertical stresses towards the side walls where friction between grains and the walls occurs: this shields the lower grain layers from the weight of the upper ones so that the apparent mass $M_{\text {app }}$ measured at the bottom is lower than the total mass $M$ of the grains.

In a recent paper [17] we demonstrated that, for a constant relative humidity $H=50 \%$, Janssen's predictions remain valid for a grain packing inside a vertical tube moving upwards relative to the grains at velocities between $10^{-2}$ and $35 \mathrm{~mm} \mathrm{~s}^{-1}$. The present study uses these results as a tool to analyse the dependence of stress transmission on the relative humidity $H$ in the range $25 \% \leq H \leq 80 \%$ : $H$ will be shown to influence strongly the variations and value of the apparent mass of the grains during the tube motion. Analyzing the motion of the grains during the tube displacement and the influence of the bead diameter confirms this influence.

The experimental setup includes a vertical glass tube (length $=400 \mathrm{~mm}$, internal diameter $D=30 \mathrm{~mm}$ ) containing a mass $M$ of monodisperse glass beads (diameter $d$, density $\rho=2.53 \times 10^{3} \mathrm{~kg} \mathrm{~m}^{-3}$ ). The tube can be moved at velocities $V$ between $10^{-1}$ to $130 \mathrm{~mm} \mathrm{~s}^{-1}$ over distances of $110 \mathrm{~mm}$ with accelerations up to $0.7 \mathrm{~m} \mathrm{~s}^{-2}$. The acceleration and deceleration distances are of order $12 \mathrm{~mm}$ at the fastest velocity and of less than $10^{-1} \mathrm{~mm}$ for $V \leq 10 \mathrm{~mm} \mathrm{~s}^{-1}$. A static cylindrical piston (diameter $29 \mathrm{~mm}$ ) is inserted at the bottom of the tube without any mechanical contact (Fig. 1); it is screwed onto a sensor measuring the force applied by the packing on the piston. The apparent mass of the grains is determined up to 256 times per second with an uncertainty of $\pm 0.1 \mathrm{~g}$. The relative humidity $H$ is regulated to within $\pm 2 \%$.

Figure 2 displays variations of the apparent mass $M_{\text {app }}$ as a function of time for three different relative humidities $H=25 \%, 60 \%$ and $80 \%$ and a same diameter $d=2 \mathrm{~mm}$ of the beads. The total mass of grains is $M=300 \mathrm{~g}$ in all experiments (the packing height is $\simeq 270 \mathrm{~mm}$ ). As expected from Janssen's model, the apparent mass after filling the tube [domain (1) in Fig. 2] is lower than $M$. Its value $M_{\text {init }}$ varies strongly from one experiment to another (typ. $\pm 50 \%$ of the mean value) due to the variability of the force chains redirecting the weight towards the side walls.

\footnotetext{
* Present address: Center for Nonlinear Phenomena and Complex Systems, and Microgravity Research Center, Université Libre de Bruxelles, Campus Solbosch, CP 165/62, 1050 Brussels, Belgium

† Present address: Laboratoire LMPDI, UMR 8108, Cité Descartes, Bâtiment Lavoisier, Université de Marne la Vallée, 77454 Marne la Vallée Cedex 2, France
} 


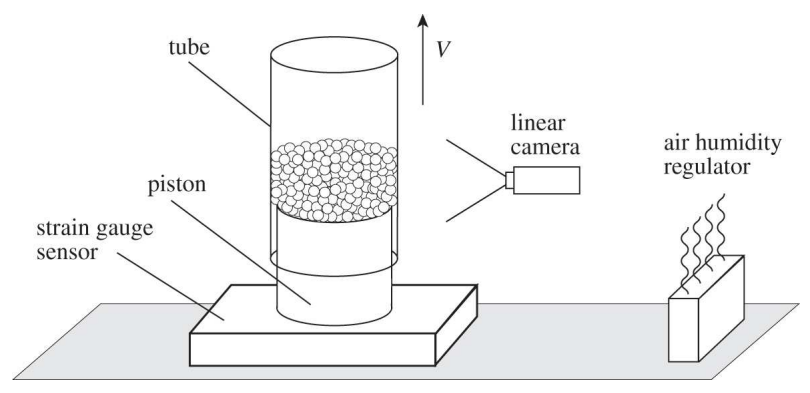

FIG. 1: Schematic view of the experimental set-up.

As the tube starts to move upwards, $M_{\text {app }}$ decreases suddenly. In contrast with the value of $M_{\text {init }}$, the variation of $M_{\text {app }}$ during the motion [domain (2) in Fig. 2] is very reproducible from one experiment to another 17] at all relative humidities $H$ (typical deviation $\pm 5 \%$ ). These variations of $M_{\text {app }}$ with time depend however on $H$ (Fig. (2).

For $H=60 \%, M_{\text {app }}$ increases towards a limiting value $M_{\mathrm{app}}^{\mathrm{dyn}}$ while the tube moves. $M_{\mathrm{app}}^{\text {dyn }}$ is reached over a distance independent of the tube velocity $V$. This result coincides with that reported previously for $H=50 \%$ [17]. The curves are similar for $H=80 \%$ (Fig. 2) and, at a given $V$, variations for $H=60 \%$ and $80 \%$ overlay well (note that the difference between the values of $M_{\text {init }}$ for $H=60 \%$ and $80 \%$ are not meaningful and correspond to the fluctuations pointed above). For $H=25 \%, M_{\text {app }}$ still drops abruptly after the tube starts to move but remains practically constant thereafter. This constant value is also larger than that of $M_{\mathrm{app}}^{\mathrm{dyn}}$ for $H=60 \%$ and $80 \%$.

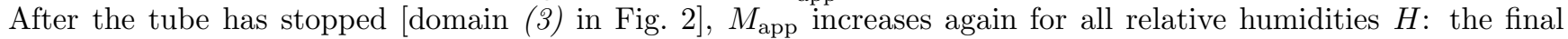
apparent mass $M_{\text {final }}$ remains however lower than the initial one $M_{\text {init }}$ and the dispersion of its values is significantly larger than in domain (2).

These results indicate that lowering $H$ influences the dynamics of the rearrangement of the force chains in the packing when the walls are set in motion. In order to study quantitatively these effects, we checked that Janssen's predictions were verified for all $H$ values. This was realized by studying variations of the limiting value $M_{\text {app }}^{\text {dyn as a }}$ function of the total mass $M$. The model assumes that the redirection of the vertical stress $\sigma_{z z}$ into an horizontal component $\sigma_{r r}$ is characterized by a coefficient $K$ and that the ratio between the tangential and normal stresses at the walls is the Coulomb friction coefficient $\mu$. Assuming a zero vertical stress $\sigma_{z z}$ at the surface of the packing $(z=0)$, its value $\sigma_{z z}\left(z_{0}\right)$ at the bottom $\left(z=z_{0}\right)$ verifies:

$$
\sigma_{z z}\left(z_{0}\right)=\rho \operatorname{cg} \lambda\left(1-e^{-z_{0} / \lambda}\right)
$$

in which the characteristic length $\lambda$ is given by:

$$
\lambda=D / 4 \mu K
$$

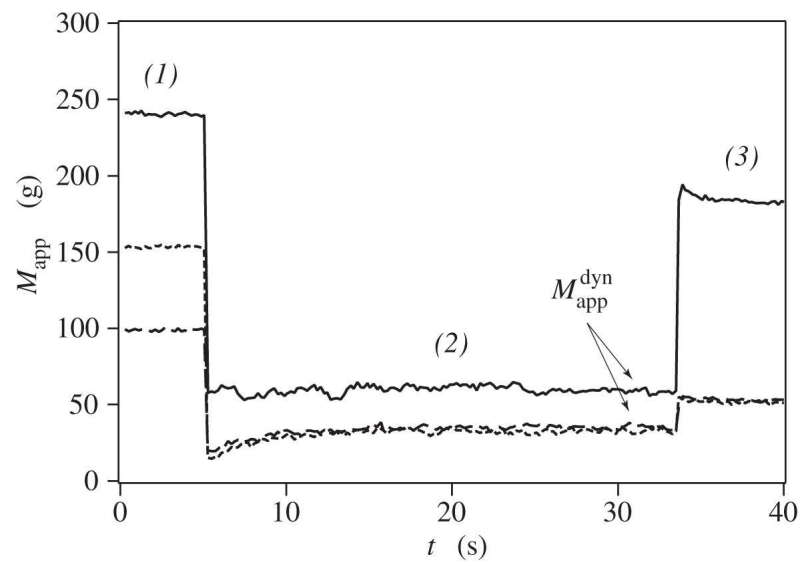

FIG. 2: Variation of the apparent mass $M_{\text {app }}$ of a grain packing $(d=2 \mathrm{~mm})$ of total mass $M=300 \mathrm{~g}$ as a function of time: (1) right after filling the tube, (2) tube moving upwards $\left(V=4 \mathrm{~mm} \mathrm{~s}^{-1}\right)$, (3) tube stopped. The curves correspond to three different relative humidities: (-) $H=25 \%,(--) H=60 \%$, (- - ) $H=80 \%$. 

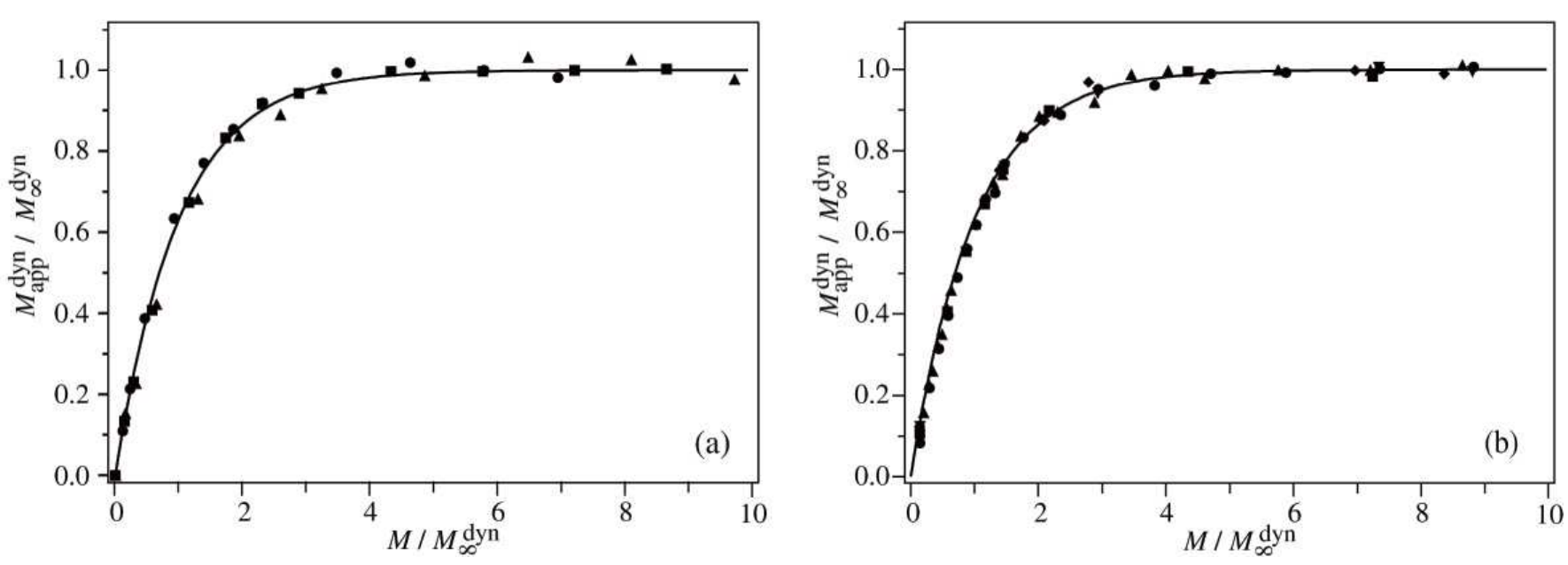

FIG. 3: Reduced apparent mass $M_{\mathrm{app}}^{\mathrm{dyn}} / M_{\infty}$ vs. reduced total mass $M / M_{\infty}$ (bead diameter $d=2 \mathrm{~mm}$ ). The solid line corresponds to Eq. (3) - (a) Tube velocity: $V=4 \mathrm{~mm} \mathrm{~s}^{-1}$, relative humidity: $(\bullet) H=25 \%,(\mathbf{\square}) H=60 \%,(\boldsymbol{\Delta}) H=80 \%$. - (b) Relative humidity: $H=60 \%$, tube velocity: $(\bullet) V=0.02 \mathrm{~mm} \mathrm{~s}^{-1},(\mathbf{\square}) V=0.2 \mathrm{~mm} \mathrm{~s}^{-1},(\bullet) V=2 \mathrm{~mm} \mathrm{~s},(\boldsymbol{\Delta}) \mathrm{V}=$ $20 \mathrm{mms}^{-1},(\mathbf{\nabla}) \mathrm{V}=34 \mathrm{~mm} \mathrm{~s}^{-1}$.

The effective weight $M_{\mathrm{app}} g$ measured by the sensor is equal to $\sigma_{z z}\left(z_{0}\right) \pi D^{2} / 4$ and the total mass $M$ of the grains is: $\rho c z_{0} \pi D^{2} / 4$ ( $c$ is the solid volume fraction of the packing). By combining Eqs. (11) and (2), one obtains the following expression of the apparent mass $M_{\text {app }}$ :

$$
\frac{M_{\mathrm{app}}}{M_{\infty}}=1-\exp \left(-\frac{M}{M_{\infty}}\right)
$$

where $M_{\infty}$ is the predicted asymptotic value of $M_{\text {app }}$ when $M$ increases:

$$
M_{\infty}=\rho c \lambda \pi D^{2} / 4 .
$$

In order to verify the validity of relation (3), the ratio $M_{\mathrm{app}}^{\mathrm{dyn}} / M_{\infty}^{\mathrm{dyn}}$ is plotted in Figs. $3 \mathrm{a}-\mathrm{b}$ as a function of the reduced total mass $M / M_{\infty}^{\mathrm{dyn}}\left(M_{\infty}^{\mathrm{dyn}}\right.$ is taken equal to the limiting value of $M_{\mathrm{app}}^{\mathrm{dyn}}$ for large values of $\left.M\right)$. Both for different relative humidities $H$ (Fig. [3 ) and different tube velocities $V$ (Fig. 3b), the variations of $H$ coincide precisely with theoretical predictions from Eq. (3).

Following these results, Janssen's hypothesis can be assumed to be valid in all our experiments. For given velocity, relative humidity and bead diameter values, a single experiment using a large total mass $M$ is required to determine the length $\lambda$ : $\lambda$ can indeed be computed from Eq. (4) using the asymptotic apparent mass $M_{\infty}^{\text {dyn }}$ measured in this way. The relative error on the value of $\lambda$ is of the order of $\pm 4 \%$.

The influence of the relative humidity is then characterized by comparing the variations of $\lambda$ with the tube velocity for three different values of $H$ (Fig. 目). For $H=60 \%$ and $80 \%, \lambda$ is nearly constant and close to the tube diameter $D$ with $\lambda=(32 \pm 1.5) m m=1.1 D$ over a range of velocities of three decades. On the contrary, for $H=25 \%, \lambda$ decreases roughly linearly with $V$ from 65 to $35 \mathrm{~mm}$ over the same range of velocities (in this curve, data points for $V \simeq 50 \mathrm{~mm} \mathrm{~s}^{-1}$ could not be obtained due to a mechanical resonance of the set-up).

Another important parameter is the diameter $d$ of the beads: variations of $\lambda$ as a function of $V$ are displayed in Fig. 40 for two different relative humidities $(H=25 \%$ and $H=80 \%)$ and four different diameters $d$ from 1.5 to $4 \mathrm{~mm}$. At a given relative humidity $H$, similar variations of $\lambda$ with the tube velocity $V$ are observed for all bead diameters. For $H=80 \%, \lambda$ is constant or varies slowly with $V$, while it decreases by a factor of 2 for $H=25 \%$. However, at $H=25 \%$, the variation is slower for the smallest beads $(d=1.5 \mathrm{~mm})$ than for the others.

Variations of the normalized apparent mass $M_{\mathrm{app}} / M_{\mathrm{app}}^{\mathrm{dyn}}$ with the normalized displacement $z / D$ provide additional information. Overall, the same qualitative differences between $H=80 \%$ and $25 \%$ are observed for all bead diameters as in Fig. 2 for $d=2 \mathrm{~mm}$. For $H=80 \%$, after a sharp initial drop, $M_{\text {app }}$ increases slowly towards a limiting value at nearly all velocities, while it remains roughly constant for $H=25 \%$. For the intermediate value $H=60 \%$, the

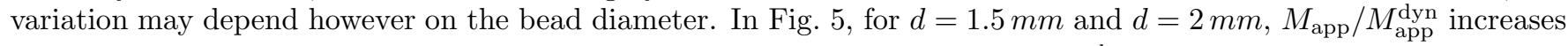
with $z / D$ towards 1 . For the largest beads $(d=4 \mathrm{~mm})$, on the contrary, $M_{\mathrm{app}} / M_{\mathrm{app}}^{\mathrm{dyn}}$ remains roughly constant while, for $d=3 \mathrm{~mm}$, the variation is intermediate. These observations may indicate that, for $H=60 \%$, small beads are 

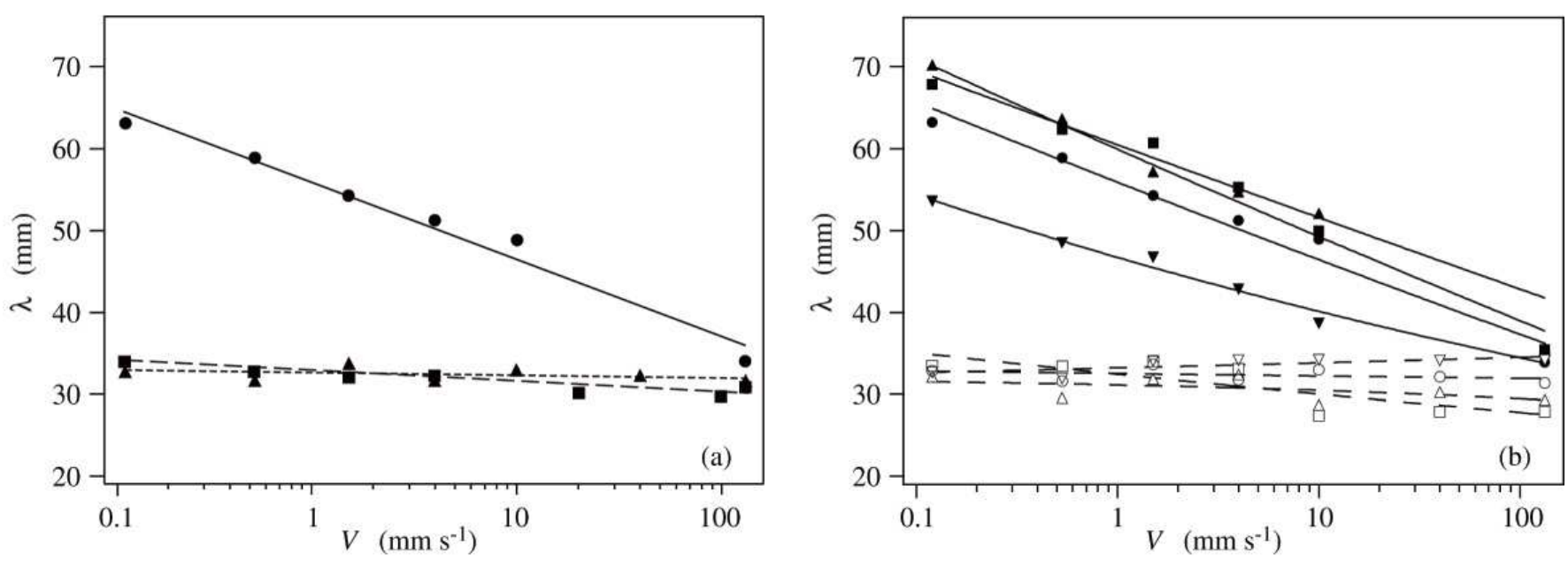

FIG. 4: Variations of Janssen's length $\lambda$ with the tube velocity $V-$ (a) for relative humidities: $(\bullet) H=25 \%,(\boldsymbol{\square}) H=60 \%$, (ム) $H=80 \%$ and bead diameter $d=2 \mathrm{~mm}$. - (b) for relative humidities $H=25 \%$ (black symbols) and $H=80 \%$ (open symbols) and for several beads diameters: $(\nabla, \mathbf{v}) d=1.5 \mathrm{~mm},(\circ, \bullet) d=2 \mathrm{~mm},(\square, \boldsymbol{\square}) d=3 \mathrm{~mm},(\triangle, \mathbf{\Lambda}) d=4 \mathrm{~mm}$.

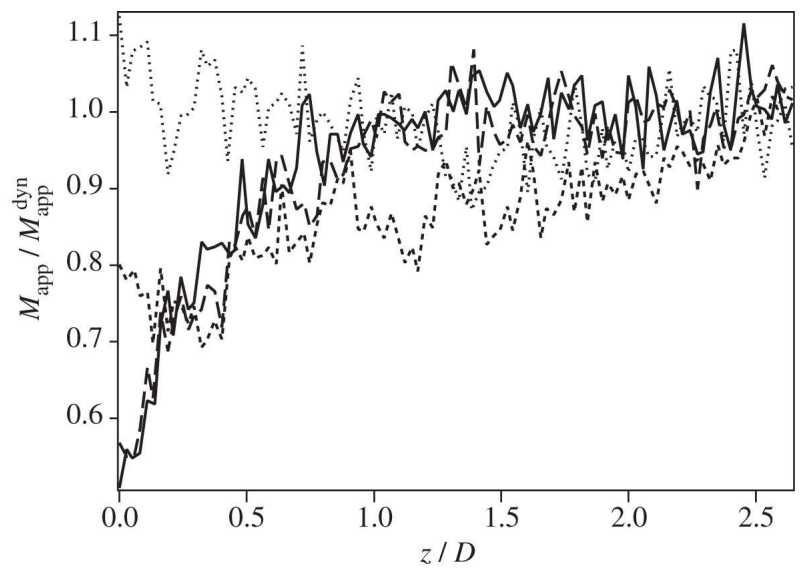

FIG. 5: Variation of $M_{\mathrm{app}} / M_{\mathrm{app}}^{\mathrm{dyn}}$ with $z / D$ for a tube velocity $V=4 \mathrm{~mm} \mathrm{~s}^{-1}$, a relative humidity $H=60 \%$ and bead diameters: $(-) d=1.5 \mathrm{~mm},(--) d=2 \mathrm{~mm},(--) d=3 \mathrm{~mm},(\cdots) d=4 \mathrm{~mm}(M=300 \mathrm{~g})$.

initially dragged upwards by the motion of the tube and that rearrangements of the packing occur later. For larger, heavier beads, this effect is smaller.

Additional clues are provided by video recordings and spatio-temporal diagrams of the bead motion close to the wall. At low relative humidities, they are almost motionless while, at large ones, they move permanently. The amplitude of the motion is larger for smaller beads and at high velocities and, in the transition regimes, in the upper part of the tube.

Spatio-temporal diagrams of light intensity variations on the outside of the packing (Fig. 6) are realized with a linear CCD camera (sampling rate: 2000 lines/s). The $x$-axis corresponds to time and the $z$-axis to distance along a vertical line in the packing. Figure 6 corresponds to an experiment during which significant bead motions occurred (note that the acceleration of the tube is instantaneous at the scale of the diagram). Horizontal stripes at the left and right ends of the diagram are obtained before and after the motion. At the beginning of the displacement, the upper surface of the packing rises sharply (strongly inclined stripes in the diagram) and the mean particle fraction decreases by $\Delta c \simeq 1.5 \%$. After the height of the packing has become constant, the grains still drift upwards, but slower and a compensating downwards flow takes place in the central part of the packing. Finally, after the tube has stopped, the packing surface does not move down to its original location. No clear-cut dependence of these particle fraction variations on the tube velocity was observed.

The upper curve in Fig. [6 displays the variation of $M_{\text {app }}$ with time during that same experiment: the variations of $M_{\text {app }}$ lasts a little longer than those of the mean particle fraction. This implies that rearrangements of the force distribution still occur after the decompaction has stopped. At low relative humidities $(H=25 \%)$, on the contrary, 


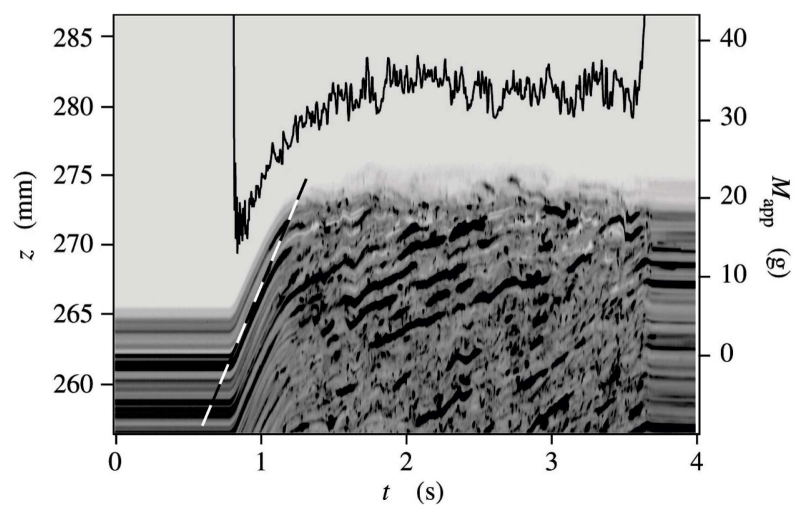

FIG. 6: Spatio-temporal diagram of the top of the grain packing ( $z=$ distance from bottom of the packing, slope of dashed line corresponds to a $25 \mathrm{~mm} \mathrm{~s}^{-1}$ velocity). Upper curve: simultaneous recording of $M_{\text {app }}$ as a function of time $(V=40 \mathrm{~mm} \mathrm{~s}$, $d=2 \mathrm{~mm}, H=60 \%)$.

no upwards drifts of the beads or of the packing surface are observed.

The results presented above demonstrate that dynamical variations of the apparent mass $M_{\text {app }}$ are very sensitive to the relative humidity $H$ although Janssen's model still remains valid. This dependence is likely to correspond to variations of the capillary forces between grains and between the grains and the walls. Many recent studies have dealt with the dependence of these forces on the content and distribution of liquids in granular materials [7, 8, [9, 21]. Even for small amounts of liquid at the contact point between beads, large capillary forces appear on a single asperity or on several asperities without requiring that pendular liquid bridges build up over the full contact region.

At large relative humidities (typ. $H=60 \%, 80 \%$ ), interactions between grains and with the walls are strong enough so that grains get dragged upwards by the tube walls (reducing strongly $M_{\mathrm{app}}$ in the process) and keep moving even after a constant value of $M_{\text {app }}$ has been reached. A significant decompaction of the packing occurs (by $2.5 \%$ in our experiments) and does not disappear after the tube has stopped. Very similar results are obtained for $H=60 \%$ and 80\%: this implies that interaction forces do not depend very much on $H$ once liquid bridges have built up on the rugosities.

For low relative humidities $(H=25 \%)$, the capillary interactions should be weaker and take place on a few asperities: this would explain why beads remain almost motionless during the experiment although the interaction with the walls is strong enough to modify the force distribution inside the packing at the onset of the motion. Also, for single beads, the ratio of the capillary forces and the weight of the grains is likely to be larger for smaller beads, explaining the distinct variation for $d=1.5 \mathrm{~mm}$ in Fig. 40. The smaller amplitude of the displacements of the beads allows in addition to reach faster a dynamical equilibrium configuration: this is shown by the time dependence of $M_{\text {app }}$ which becomes immediately constant in contrast with higher values of $H$. Also, for $H=25 \%$, the perturbation induced by the motion increases with the final velocity $V$ of the tube: this is shown by the linear decrease of $M_{\mathrm{app}}^{\text {dyn }}$ with $V$ and contrasts with its constant value for $H=60 \%$ and $80 \%$.

Note that no electrostatic effects were observed, even for $H=25 \%$.

To conclude, these experiments have allowed for a sensitive study of the dependence of force screening effects on the relative humidity. Increasing the latter enhances interactions between grains and with the walls, resulting in a significant decompaction and in a much larger amplitude of the grain motions. Janssen's model however still describes correctly in all cases variations of the effective mass with the height of the packing: the main difference is a decrease of the screening length with an increasing tube velocity for the lowest relative humidity while it is constant at larger ones. In future works, this information will need to be complemented by the influence of two additional important parameters: the roughness of the tube walls and of the beads and the mean particle fraction of the packing.

We thank Ch. Frénois for the realization and development of the motion control system, G. Chauvin and R. Pidoux for the realization of the experimental setup, É. Clément and B. Perrin for helpful discussions and Ph. Gondret for a careful reading of the manuscript.

[1] S. Laouar and Y. Molodtsof. Experimental characterization of the pressure drop in dense phase pneumatic transport at very low velocity. Powder Technol., 95:165-173, 1998. 
[2] Y. Bertho, F. Giorgiutti-Dauphiné, T. Raafat, E. J. Hinch, H. J. Herrmann, and J. -P. Hulin. Powder flow down a vertical pipe: the effect of air flow. J. Fluid Mech., 459:317-345, 2002.

[3] Y. Bertho, F. Giorgiutti-Dauphiné, and J. -P. Hulin. Intermittent dry granular flow in a vertical pipe. Phys. Fluids, 15(11):3358-3369, 2003.

[4] R. Albert, I. Albert, D. J. Hornbaker, P. Schiffer, and A.-L. Barabási. Phys. Rev. E, 56:R6271, 1997.

[5] L. Bocquet, É. Charlaix, S. Ciliberto, and J. Crassous. Moisture-induced ageing in granular media and the kinetics of capillary condensation. Nature, 396:735-737, 1998.

[6] N. Fraysse, H. Thomé, and L. Petit. Humidity effects on the stability of a sandpile. Eur. Phys. J. B, 11:615-619, 1999.

[7] D. J. Hornbaker, R. Albert, I. Albert, A. L. Barabási, and P. Schiffer. What keeps sandcastles standing? Nature, 387:765, 1997.

[8] T. G. Mason, A. J. Levine, D. Ertas, and T. C. Halsey. Critical angle of wet sandpiles. Phys. Rev. E, 60(5):R5044-R5047, 1999.

[9] P. Tegzes, R. Albert, M. Paskvan, A. L. Barabási, T. Vicsek, and P. Schiffer. Liquid induced transition in a granular media. Phys. Rev. E, 60(5):5823-5826, 1999.

[10] J. -Ph. Bouchaud, M. E. Cates, and Ph. Claudin. Stress distribution in granular media and nonlinear wave equation. J. Phys. I France, 5:639-656, 1995.

[11] C. C. Mounfield and S. F. Edwards. A theoretical model for the stress distribution in granular matter. ii. forces in pipes. Physica A, 226:12-24, 1996.

[12] D. M. Mueth, H. M. Jaeger, and S. R. Nagel. Force distribution in a granular medium. Phys. Rev. E, 57(3):3164-3169, 1998.

[13] E. B. Pitman. Forces on bins: the effect of random friction. Phys. Rev. E, 57(3):3170-3175, 1998.

[14] L. Vanel, Ph. Claudin, J. -Ph. Bouchaud, M. E. Cates, É. Clément, and J. P. Wittmer. Stresses in silos: comparison between theoretical models and new experiments. Phys. Rev. Lett., 84(7):1439-1442, 2000.

[15] G. Ovarlez, É. Kolb, and É. Clément. Rheology of a confined granular material. Phys. Rev. E, 64:060302, 2001.

[16] G. Ovarlez, C. Fond, and É. Clément. Overshoot effect in the janssen granular column: a crucial test for granular mechanics. Phys. Rev. E, 67:060302R, 2003.

[17] Y. Bertho, F. Giorgiutti-Dauphiné, and J. -P. Hulin. Dynamical janssen effect on granular packing with moving walls. Phys. Rev. Lett., 90(14):144301, 2003.

[18] J. W. Landry, G. S. Grest, L. E. Silbert, and S. J. Plimpton. Confined granular packings: structure, stress, and forces. Phys. Rev. E, 67:041303, 2003.

[19] J. W. Landry and G. S. Grest. cond-mat/0309471 2003.

[20] H. A. Janssen. Versuche über getreidedruck in silozellen. Z. Ver. Dtsch. Ing., 39:1045-1049, 1895.

[21] T. C. Halsey and A. J. Levine. How sandcastles fall. Phys. Rev. Lett., 80(14):3141-3144, 1998. 